\title{
Adaptive Sleep Scheduling for Health Monitoring System Based on the IEEE 802.15.4 Standard
}

\author{
Nurul Fahmi, M. Udin Harun Al Rasyid, Amang Sudarsono \\ Graduate School of Informatics and Computer Engineering \\ Politeknik Elektronika Negeri Surabaya \\ Jl. Raya ITS Sukolilo Surabaya 60111, Indonesia \\ Telp: 62315947280 Fax : 62315946114 \\ E-mail: nurulfahmi@pasca.student.pens.ac.id, \{udinharun,amang\}@pens.ac.id
}

\begin{abstract}
In the recent years, Wireless Sensor Networks (WSNs) have become a very popular technology for research in various fields. One of the technologies which is developed using WSN is environmental health monitoring. However, there is a problem when we want to optimize the performance of the environmental health monitoring such as the limitation of the energy. In this paper, we proposed a method for the environmental health monitoring using the fuzzy logic approach according to the environmental health conditions. We use that condition to determine the sleep time in the system based on IEEE 802.15.4 standard protocol. The main purpose of this method is to extend the life and minimize the energy consumption of the battery. We implemented this system in the real hardware test-bed using temperature, humidity, $\mathrm{CO}$ and $\mathrm{CO} 2$ sensors. We compared the performance without sleep scheduling, with sleep scheduling and adaptive sleep scheduling. The power consumption spent during the process of testing without sleep scheduling is 52\%, for the sleep scheduling is $13 \%$, while using the adaptive sleep scheduling is around $7 \%$. The users also can monitor the health condition via mobile phone or web-based application, in real-time anywhere and anytime.
\end{abstract}

Keywords: WSN, Environmental Health Monitoring, Adaptive Sleep Scheduling, Fuzzy Logic.

\section{INTRODUCTION}

Internet of Things (IoT), the new communication technology is rapidly developing. This phenomenon is indicated by the massive used of technology for various kinds of knowledge. This has caused the change of technology transition. In the end, this technology can help people to solve the issue in many fields. One of the new technology that very popular at the moment is Wireless Sensor Network (WSN). This WSN has the ability to do a sensor computing and a communication process with other devices efficiently. 
Environmental health is an ecological junction between people and environments which is aimed to increase the quality of life, focusing on the health and the happy human life. Environmental health affects the smoothness of human life because the environment is a human place to live. Recently, WSN technology has been widely used in many important things. This is because each sensor node of WSN has many advantages such as low power consumption, low implementation cost, large coverage and can be applied in real-time [1]. There are many WSN technologies that have been used and applied recently, such as e-health system, military system, agriculture sensor system, forest fire monitoring system, smart home automation, smart city sensor, and environmental health monitoring system [2]. In the environmental health monitoring, for example, the sensor nodes can be helpful in the environmental health to monitor the condition and also can provide the information whether the condition is good or bad. In the field of e-health, for example, many researchers conducted a study to monitor the health of the human body, such as monitoring pulse oximetry of body temperature, heartbeat, and oxygen in the human blood.

Each sensor node that we used has several components in it such as Waspmote Board, Sensor Board, Sensors, ZigBee, and also battery [14]. The most exciting challenges in this research are the battery consumption that used by each sensor node because the lifetime of sensor node depends on the battery. From the data sensing, idle, and transmission data process, the most wasteful power consumption is at the moment when it comes to transmitting the data.

For minimizing the problem above, we propose the fuzzy logic approach to process the adaptive transmission from the sensor node to the gateway. This is aimed to save the battery consumption. In this paper, we compare three processes of data transmission process in WSN that aiming to reduce the battery consumption. First, we start from the system without using sleep scheduling. Second, we use sleep scheduling, and the last, we use the adaptive sleep scheduling where the sensor node will transmit the data in a set time to monitor the environmental health based on the output of fuzzy logic.

In addition, to save power consumption of the battery, we also propose the monitoring system of real-time environmental health condition where each of the sensor nodes send the data to the gateway through IEEE 802.15.4 standard protocol to store the data temporarily. The sensors which are used in this system are temperature, humidity, carbon monoxide (CO) and carbon dioxide (CO2) sensors. Furthermore, the sensor data is collected at the gateway and will be synchronized to Data Center Server (DCS) through Local Area Networks (LANs) to save in DCS database permanently. All users can access DCS by using a mobile phone or web-based application in real-time anywhere and anytime. 
This research paper is organized as follows, The Section 2, shows the related works. The Section 3, we describe the originality of our paper. The Section 4, we describe the fundamental of fuzzy logic and system design of our environmental health monitoring. The Section 5, we propose an adaptive sleep scheduling method based on the fuzzy logic approach. The Section 6 presents the experiment and analyzes the power consumption in real hardware implementation. Finally, we explain a conclusion in Section 7.

\section{RELATED WORKS}

Recently, many researchers have been researched about the WSN technology for environmental health monitoring and for saving the energy. Ferdoush, et al. [1] present the survey about WSN that is applied in the environmental field monitoring system, such as in agriculture monitoring, habitat monitoring, indoor living monitoring, greenhouse monitoring, climate monitoring, and forest monitoring. Othmana et al. [2] proposed a design and implementation of environmental health condition monitoring system based on WSN using open-source hardware platforms, such as Raspberry Pi and Arduino microcontroller. The Othmana's system [2] used temperature and humidity sensors to monitor the temperature and humidity value. For communication between the sensor node and the gateway (Raspberry Pi), Othmana used Xbee module 802.15.4 standard protocol.

Ye, et al. [3] proposed an environmental health monitoring by using several sensors such as underground water level, barometric pressure, ambient temperature, atmospheric humidity, wind direction, wind speed and a rainfall sensor. Ye proposed the WSN architecture of the node hardware, data acquisition, data processing with gateway and data visualization. In this system, they used IRIS mode hardware platform, where communication between base-station with a gateway is applied via internet or GPRS. All users can monitor the results in real-time via a web-based application. In paper [4,5], the researchers proposed Indoor Air Quality monitoring which is developed using Arduino, Xbee 802.15.4 standard protocol for communication and micro gas sensor. This system used many sensors such as CO2, Volatile Organic Compounds (VOC), temperature, and humidity sensor. Base Station (BS) receives the data from each sensor node periodically, then the data is saved in the database as data storage and also data management.

From the research that has been done [1-5], the researchers only did the measurement to know the environmental health condition. Actually, it already could help humans, but this could not give the decision whether this condition is good or bad. Many methods are being made to take a decision on many problems, one of the decision-making method is a fuzzy logic approach. Dutta, et al. [6] proposed the system for detecting forest fire using WSN based on the fuzzy logic approach. This system used MATLAB simulation to detect the probability of a forest fire. 
Fahmi, et al. [7] proposed a fuzzy logic approach for an implementation of environmental health monitoring based on WSN. The sensor used in that research is temperature, humidity, $\mathrm{CO}$ and $\mathrm{CO} 2$ sensors. The communication between the sensor node and the gateway use ZigBee 802.15.4 standard protocol. This system is applied to real hardware by using Microcontroller ATMega 1281 and Gases Sensor Board developed by Waspmote [15].

Tripathy, et al. [8] analyzed an energy efficiency of fuzzy logic based on the intelligent WSN. This experiment has been done in fuzzy logic toolbox inside the MATLAB simulation software. For reducing the power consumption, they applied a sensor node in sleep mode when there is no activity to transmit data. And also they used the scheme based on the combination of a hierarchical and cluster-based network. Petri net modeling and HPSim simulator are used as a hybrid topology network which is aimed to analyze, verify correlation of the sensor node and test every state using ON/OFF mode for reducing power consumption.

Giovanni Pau. [9] proposed a novel energy efficiency of power consumption reduction by using the fuzzy logic approach in accordance with the battery level and Ratio Throughput for determining sleep time from a sensor node based on IEEE 802.15.4 standard protocol. This system used 3 memberships value such as sleeping time, Ratio of Throughput and battery level. This simulation is done by using the Fuzzy Logic Control (FLC) proposed to reduce battery power consumption.

Sabitha, et al. [10] combined two algorithms which are Neural Network (NN) and Fuzzy Logic Control (FLC) for saving power consumption using soft computing based on the transmission power control techniques. Their design consists of 4 Transmission Power Consumption (TPC) algorithms which are FLC-TFC, FLC-GA, ANN-TPC, and ANN-GA-TPC. The transmission used to analyze the throughput, average power, average energy power conducted by successful transmission. The result showed that ANN-TPC outperforms FLCTPC excellently in terms of energy saving.

\section{ORIGINALITY}

The WSN technology is growing very rapidly for monitoring environmental health. In the previous research [2], they implemented the WSN technology for monitoring temperature, humidity. However, they did not discuss the power consumption of the battery. There are many algorithms to reduce the consumption of batteries, one of them is a fuzzy logic such as $[8,10]$, where they analyzed the power energy consumption using Matlab simulations and mathematical calculations. They did not prove it in a real hardware case. Another research that has been done [9], where the authors proposed environmental health monitoring for minimizing energy consumption with a fuzzy logic based on the battery level and to Throughput Ratio for the sleep duration.

The contribution of this paper, we implement real hardware by using the temperature, humidity, $\mathrm{CO}$ and $\mathrm{CO} 2$ sensors. To obtain environmental 
health conditions, we use the fuzzy logic approach as a decision maker. We use the output of fuzzy logic such as sleep duration and transmission control which we named adaptive sleep scheduling. Also, we provide the system for monitoring the condition of environmental health in real-time, where all users can access using their smartphone and/or web-based application anywhere and anytime.

\section{Fuzzy Logic for Environmental Health Monitoring}

This section describes the overview of fuzzy logic. Fuzzy logic is one of the fundamental building blocks of soft computing. Fuzzy logic was first introduced by Lotfi A. Zadeh in 1965 [12]. Many researchers use the fuzzy logic approach as a decision making, for example, in the field of agriculture [13], fuzzy logic is used to determine the probability of forest fire [6], and also for monitoring the health condition [7]. This method uses artificial intelligence to determine the basis of human thinking for decision making in the processing of one or many inputs. In Crisp, the membership value an item $\mathrm{x}$ in a set $\mathrm{A}$, which is often written with $\mu \mathrm{A}(\mathrm{x})$, has two possibilities, namely 1 and 0 . The value of 1 means that an item becomes a member in a set, while 0 means that an item is not a set. Fuzzy logic is divided into three, named fuzzification, fuzzy processing, and defuzzification. Figure 1 shows an architecture fuzzy logic system.

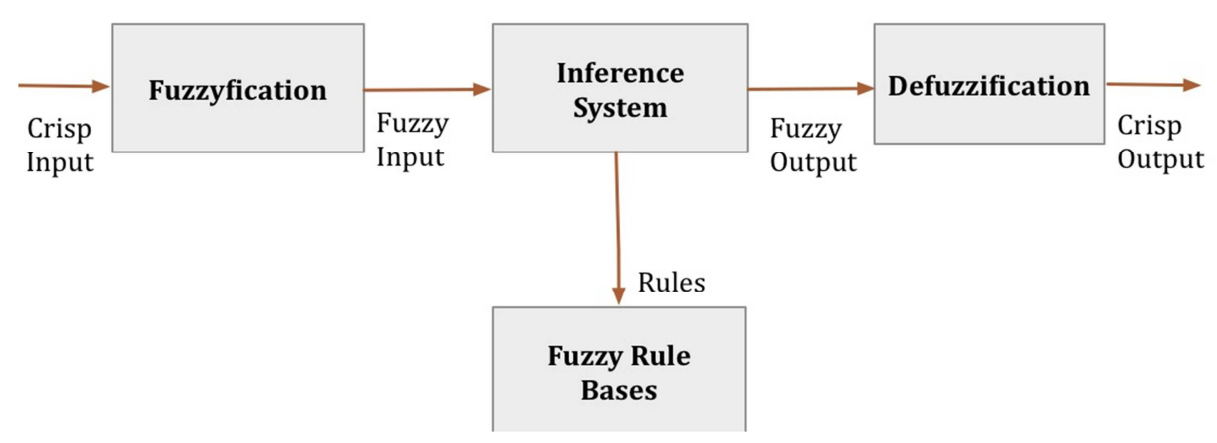

Figure 1. Architecture fuzzy logic system [13]

(a) Fuzzification : The process of changing the inputs which truth value are uncertain (crisp input) in the form of fuzzy input.

(b) Inference System : The process of analyzing and utilizing fuzzy input and fuzzy rules which have been determined. The result is fuzzy output.

(c) Defuzzification : The process of changing the fuzzy output into a crisp value based on the predetermined membership functions. 


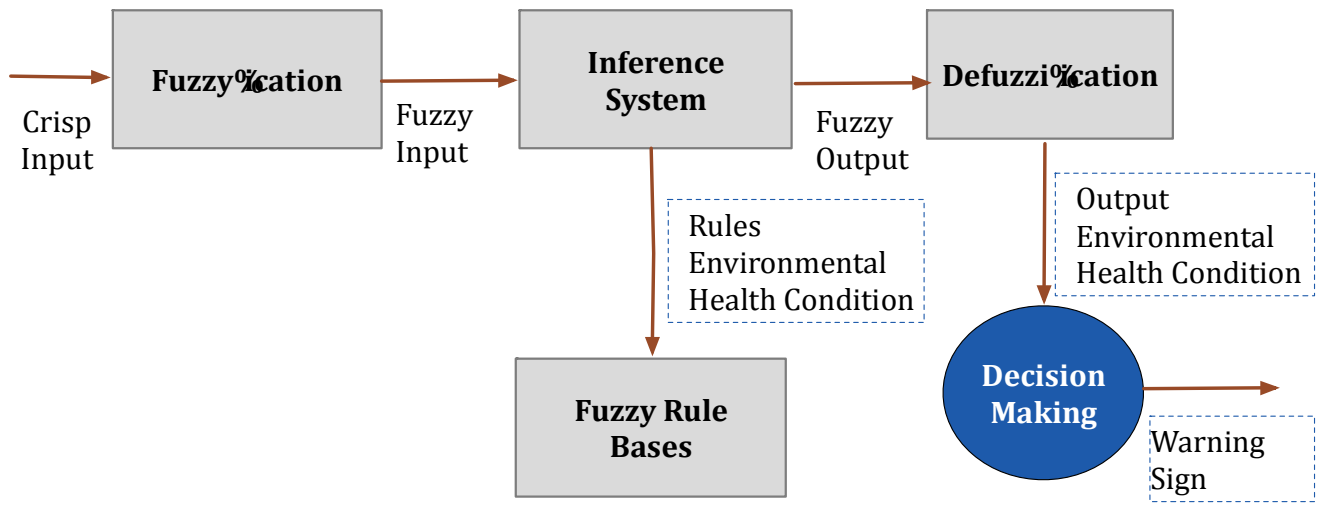

Figure 2. Proposed fuzzy logic for environmental health monitoring

Figure 2 shows the block diagram for environmental health monitoring using fuzzy logic approach. In this case, we have four membership function variables. Each membership function has three levels : Temperature (Low, Medium, High), Humidity (Low, Medium, High), CO (Low, Medium, High) and CO2 (Low, Medium, High). Data from the four sensors will be combined to an output which is called environmental health condition. The environmental health condition output level consists of five categories: Very Good (VG), Good (G), Average (A), Bad (B) and Dangerous (D).

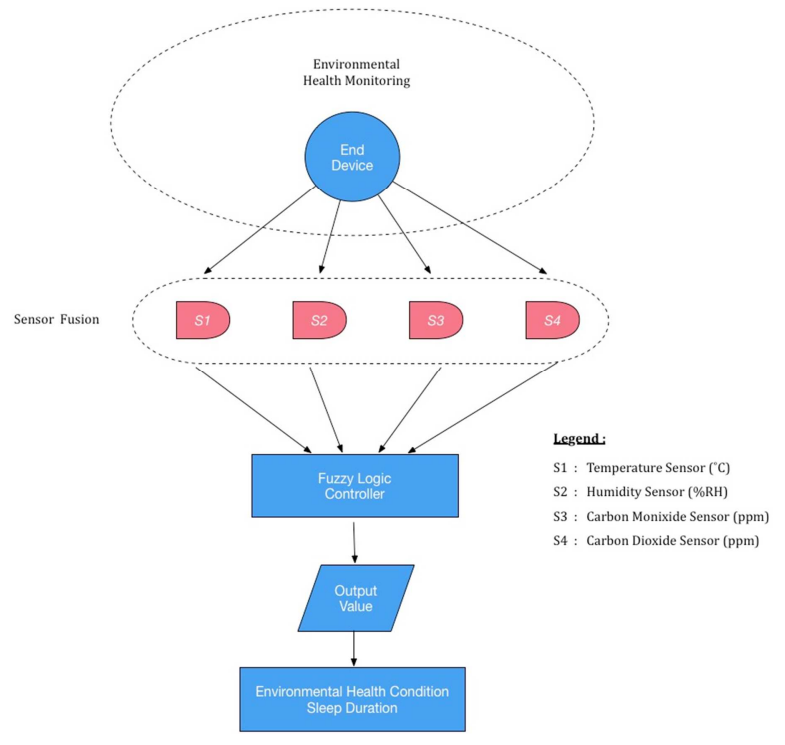

Figure 3. Design FLC data fusion

Furthermore, data from the four sensors will be fused and processed using a fuzzy logic control such as shown in figure 3. The result of the processing fuzzy logic is an environmental health condition. This condition is used for determining the sleep duration of Gases Sensor Board, ZigBee and Waspmote Board. And this is also used for transmitting the data sensor to the gateway. 


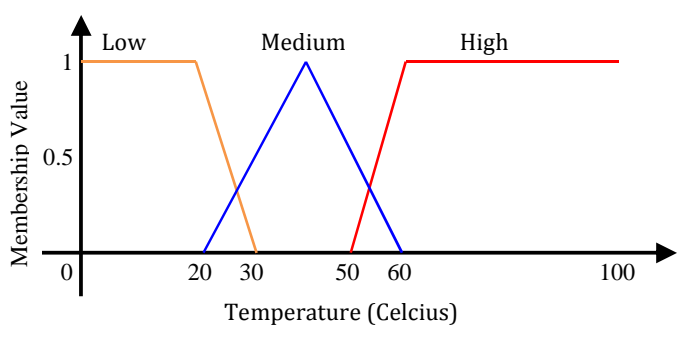

(a)

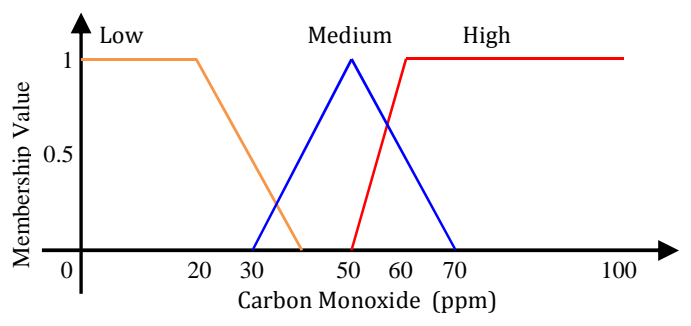

(c)

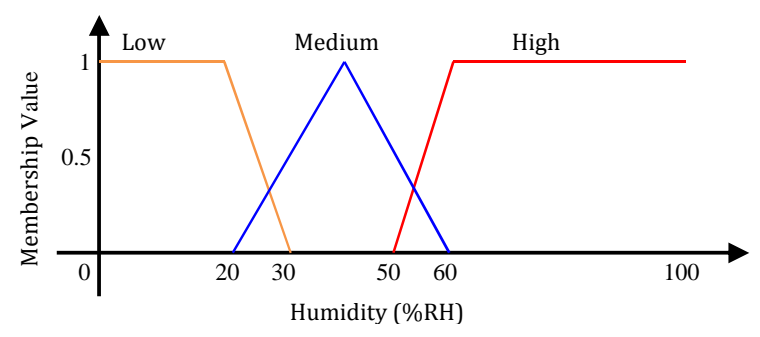

(b)

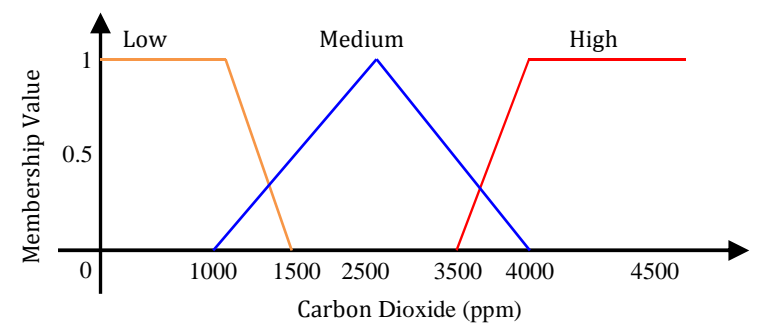

(d)

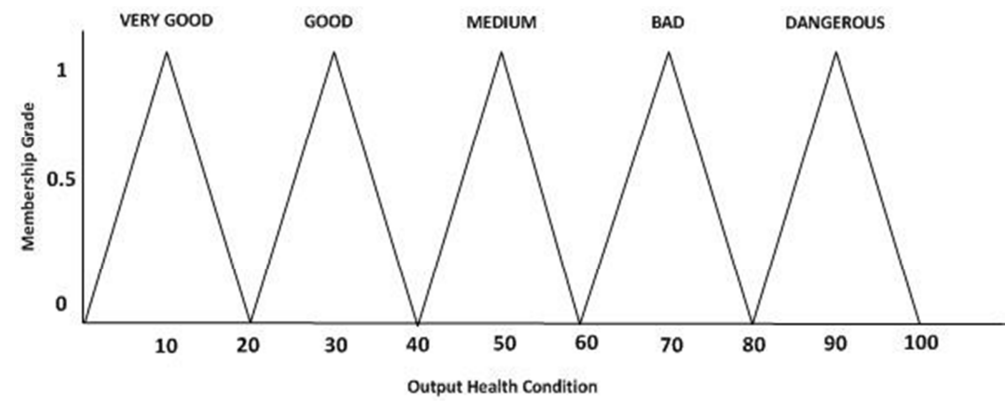

(e)

Figure 4. Membership function of fuzzy logic variable : (a) Membership function of temperature, (b) Membership function of humidity, (c) Membership function of CO, (d) Membership function of CO2, (e) The Output of environmental health condition

Figure 4 shows the membership function that serves as an input value of each variable (sensor value), these will become a value of linguistic variables that have a membership value ranging from 0 to 1 in each fuzzy set (fuzzification process). After designing the membership function of each sensor and get the value of its output, the next step is to design a fuzzy logic rule in the form of IF ... THEN. This is used as a fuzzy knowledge base. Fuzzy inference systems that are used in our work is Tsukamoto method. In general, the form Tsukamoto fuzzy model can be seen in the following simple rules:

$$
\text { If }(X \text { is } A) \text { and }(Y \text { is } B) \text { then ( } Z \text { is } C)
$$


Where $\mathrm{X}, \mathrm{Y}$, and $\mathrm{Z}$ is fuzzy variable. While $\mathrm{A}, \mathrm{B}$, and $\mathrm{C}$ are a fuzzy set of each variable. In this method, each consequent upon the rules are in the form of IF... THEN and represented in a fuzzy set. Fig 5 shows the inference system of Tsukamoto method.

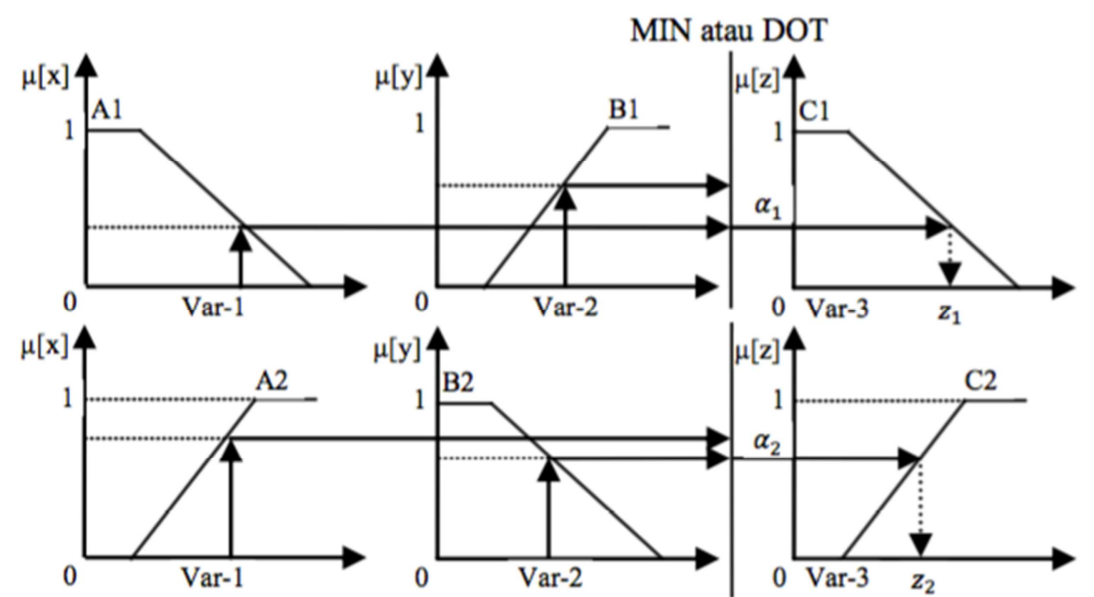

Figure 5. Fuzzy inference system Tsukamoto method [13]

Fuzzy inference system consists of several rules. This process has a membership function, logical operators, and IF-THEN rules. This method is often known as MAX-MIN. For the inference system, we used 4 input variables which consist 3 membership function, namely LOW, MEDIUM, HIGH. So, the total is $3 \times 3 \times 3 \times 3=81$ rules used in the decision making of the environmental health condition.

Table 1. Inference rules for environmental health condition

\begin{tabular}{cccccc}
\hline \hline Rule & Temperature & Humidity & CO & CO2 & $\begin{array}{c}\text { Health } \\
\text { Condition }\end{array}$ \\
\hline 1 & Low & High & Low & Low & Very Good \\
2 & Low & High & Low & Medium & Good \\
3 & Low & High & Low & High & Average \\
4 & Low & High & Medium & Low & Good \\
5 & Low & High & Medium & Medium & Average \\
6 & Low & High & Medium & Hugh & Bad \\
$\bullet \bullet$ & $\bullet \bullet \bullet \bullet \bullet$ & $\bullet \bullet \bullet \bullet \bullet$ & $\bullet \bullet \bullet \bullet \bullet$ & $\bullet \bullet \bullet \bullet \bullet$ & $\bullet \bullet \bullet \bullet \bullet$ \\
$\bullet \bullet$ & $\bullet \bullet \bullet \bullet \bullet$ & $\bullet \bullet \bullet \bullet \bullet$ & $\bullet \bullet \bullet \bullet \bullet$ & $\bullet \bullet \bullet \bullet \bullet$ & $\bullet \bullet \bullet \bullet \bullet$ \\
80 & High & Low & High & Medium & Dangerous \\
81 & High & Low & High & High & Dangerous \\
\hline
\end{tabular}

Table 1 shows the several inference rules for environmental health condition. With this rule, some variables are combined to produce the output of health conditions. If represented like the $1^{\text {st }}$ rule, IF (Temperature is Low) AND (Humidity is High) AND (CO is Low) AND (CO2 is Good) Then (Health 
Condition is Very Good). For combining some rules, "AND" operator is used in the rules of fuzzy. Defuzzification process is the process to restore the value of the degree of the fuzzy membership processing results in the form of the actual output value.

Equation (2) is a mathematical representation of a process called defuzzification. It is given by the following formula:

$$
Z=\frac{\int \mu A(z) * z d z}{\int \mu A(z) d z}
$$

Where $\mu A$ is membership function, $d z$ is membership degree with maximum values is 1 , and $Z$ is fuzzification output.

\section{Proposed Adaptive Sleep Scheduling Fuzzy Logic Control (FLC) Approach in Environmental Health Monitoring}

In this paper, we proposed adaptive sleep scheduling for monitoring environmental health condition using FLC. Figure 6 shows a proposed system for environmental monitoring condition based on WSN. First, sensor node will collect data and use ZigBee 802.15.4 standard protocol for communicating with the gateway. As a decision making, we use a fuzzy logic approach to implement the environmental health condition monitoring. This system automatically gives the decision of the measurement results in the form of warning such as Very Good (VG), Good (G), Average (A), Bad (B), and the last is Dangerous (D). In the gateway, the data from the sensor will be saved to temporary storage provided by medium with the limited storage capacity of 8GB [16]. Furthermore, the sensor data will be collected in the gateway and synchronized to the DCS via TCP / IP to store the data permanently. All users can access data sensor via web-based or mobile phone application anywhere and anytime while connected to the Internet. 


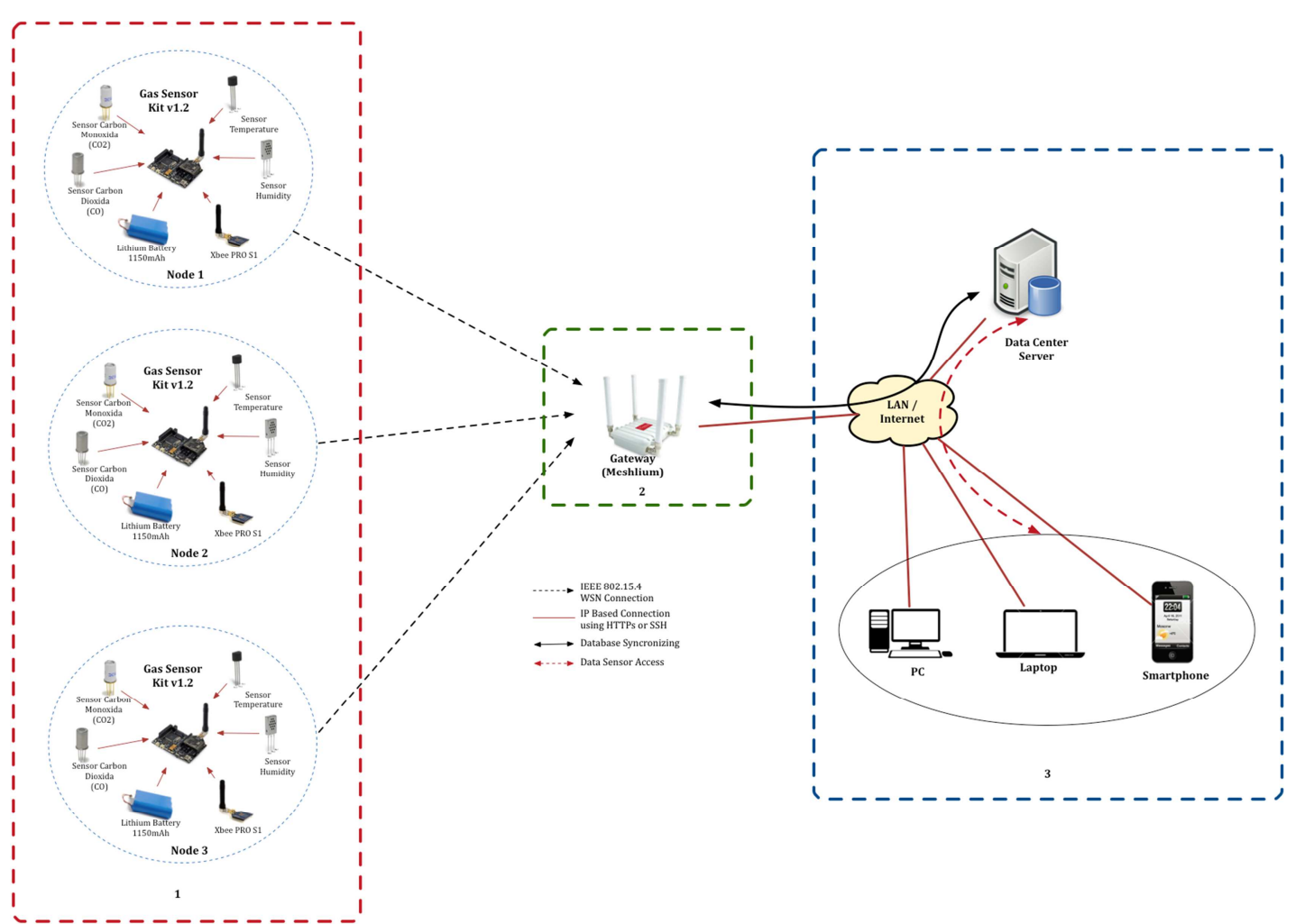

Figure 6. Proposed topology for environmental health monitoring system based on WSN

\subsection{Data Sensor Transfer and Fuzzification of Decision Making}

In this paper, we use three sensor nodes (Node1, Node2, and Node3) which are used to monitor the environmental health condition. We use four sensors such as temperature, humidity, $\mathrm{CO}$ and $\mathrm{CO} 2$ sensors, which allow the inclusion of all sensors at the same time and power using lithium battery with 3.7 Volt and the capacity of $1150 \mathrm{mAh}$. And also for communicating between sensor node to the gateway, ZigBee 802.15.4 protocol standard is applied. Here, sensor node will measure the data of the sensors. From the measurement results above, all of the sensor data will be combined into one to make a decision using a fuzzy logic method. The goal is to know the environmental health condition and sleep scheduling for the control of transmission data to a gateway based on environmental health condition such as shown figure 7 . 


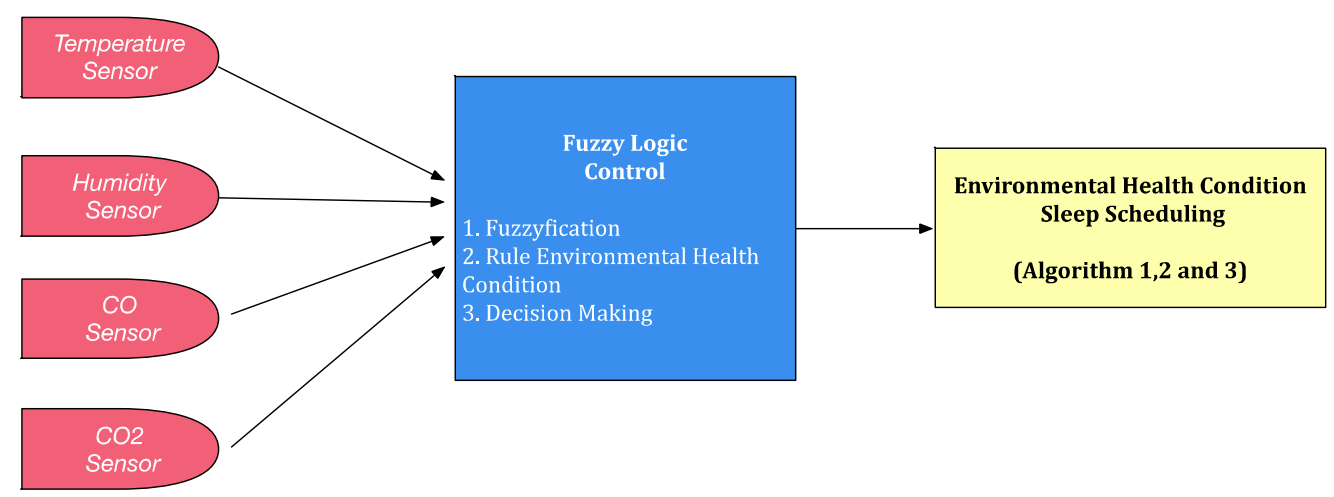

Figure 7. Fuzzification for the decision making

The output of environmental health condition is used as transmission control for sleep scheduling which is aimed to reduce the battery consumption. In this case, The fuzzy logic approach without sleep scheduling is shown in Algorithm 1. Furthermore, an algorithm with sleep scheduling is shown in Algorithm 2. We proposed the system which every 2 minutes sensor node will go to sleep and after that sensor node will wake up and sensing the data again.

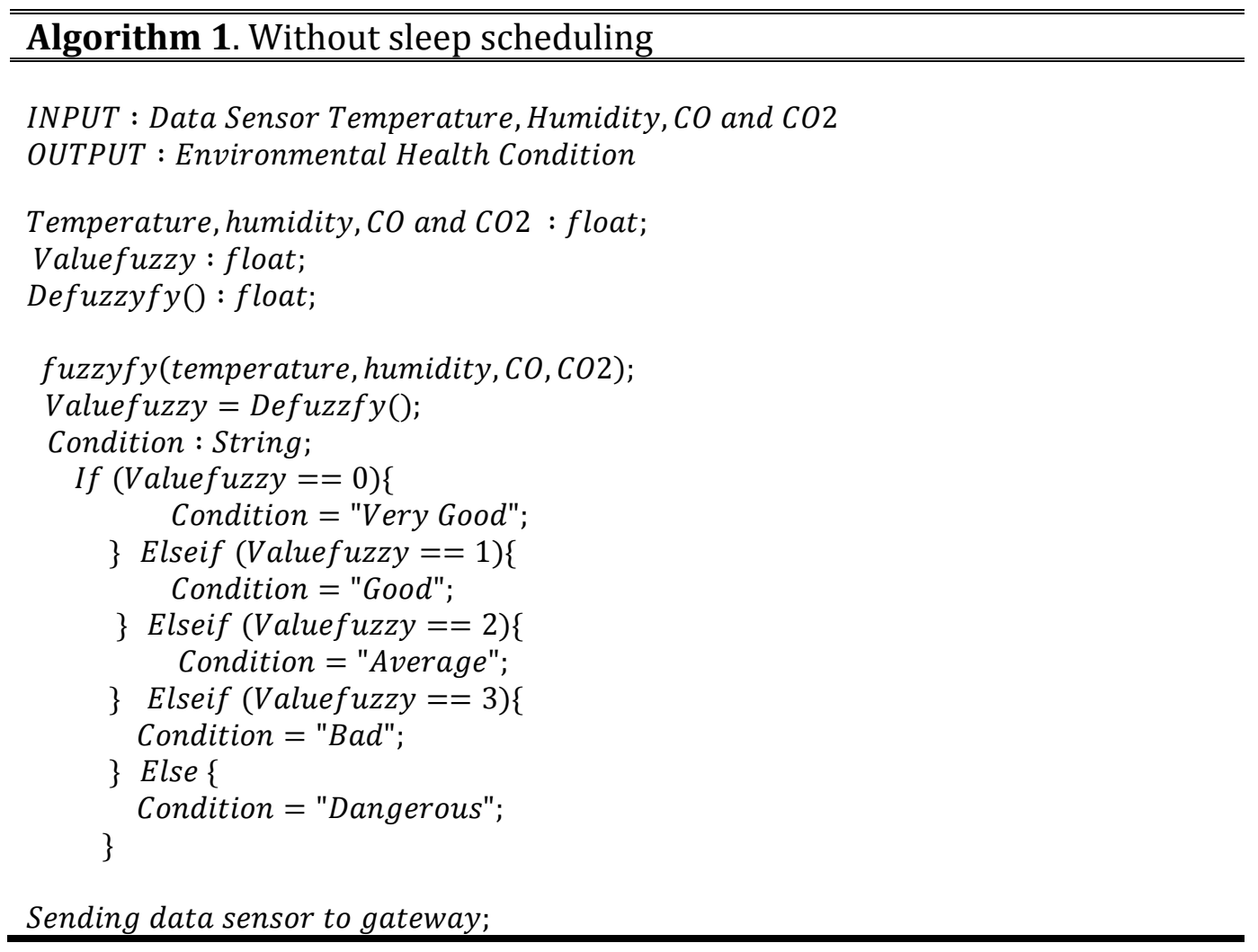




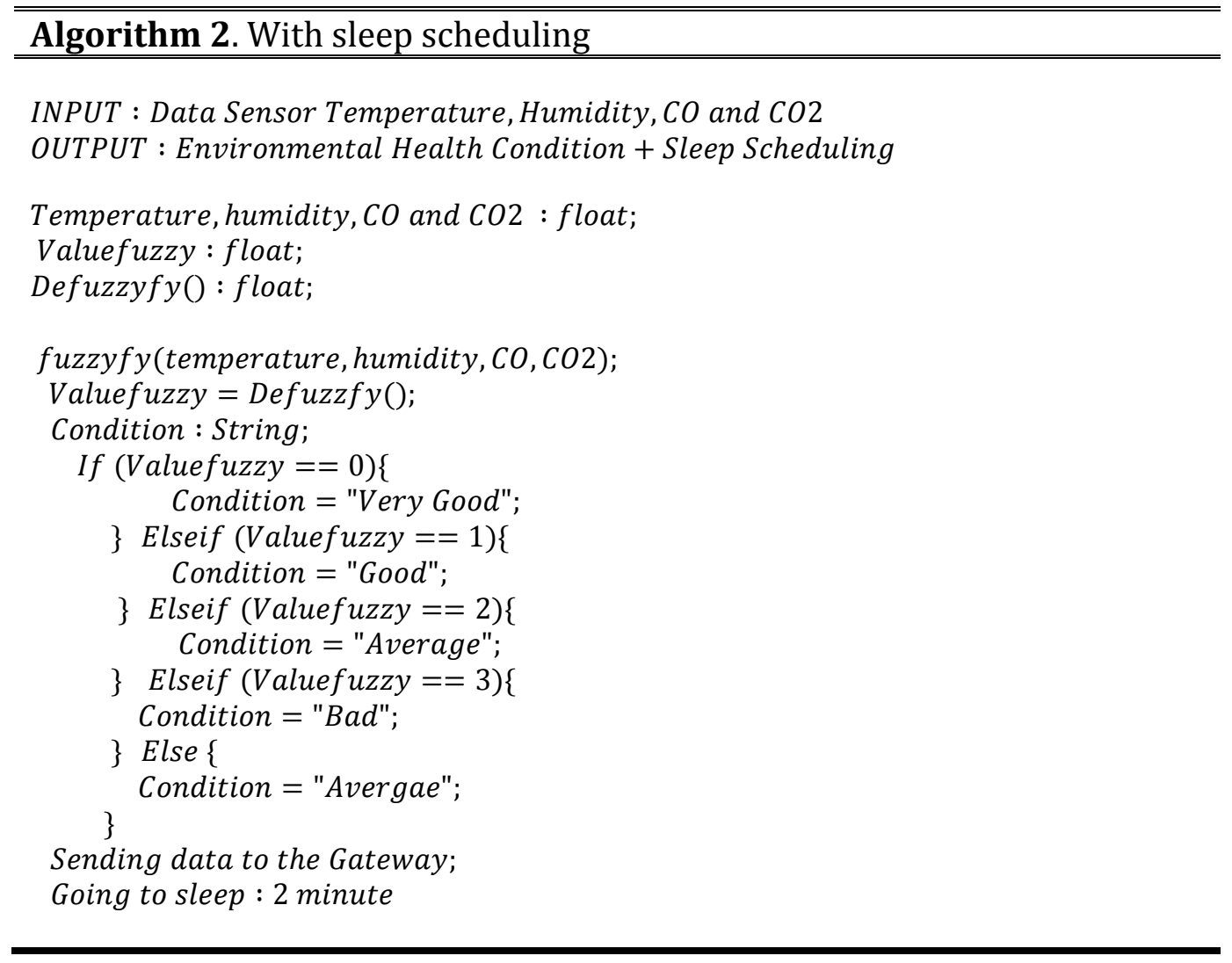

And the last, we propose an adaptive sleep scheduling algorithm as shown in Algorithm 3. We proposed transmission control to schedule the sleep of the sensor node based on the output of environmental health condition using a fuzzy logic approach.

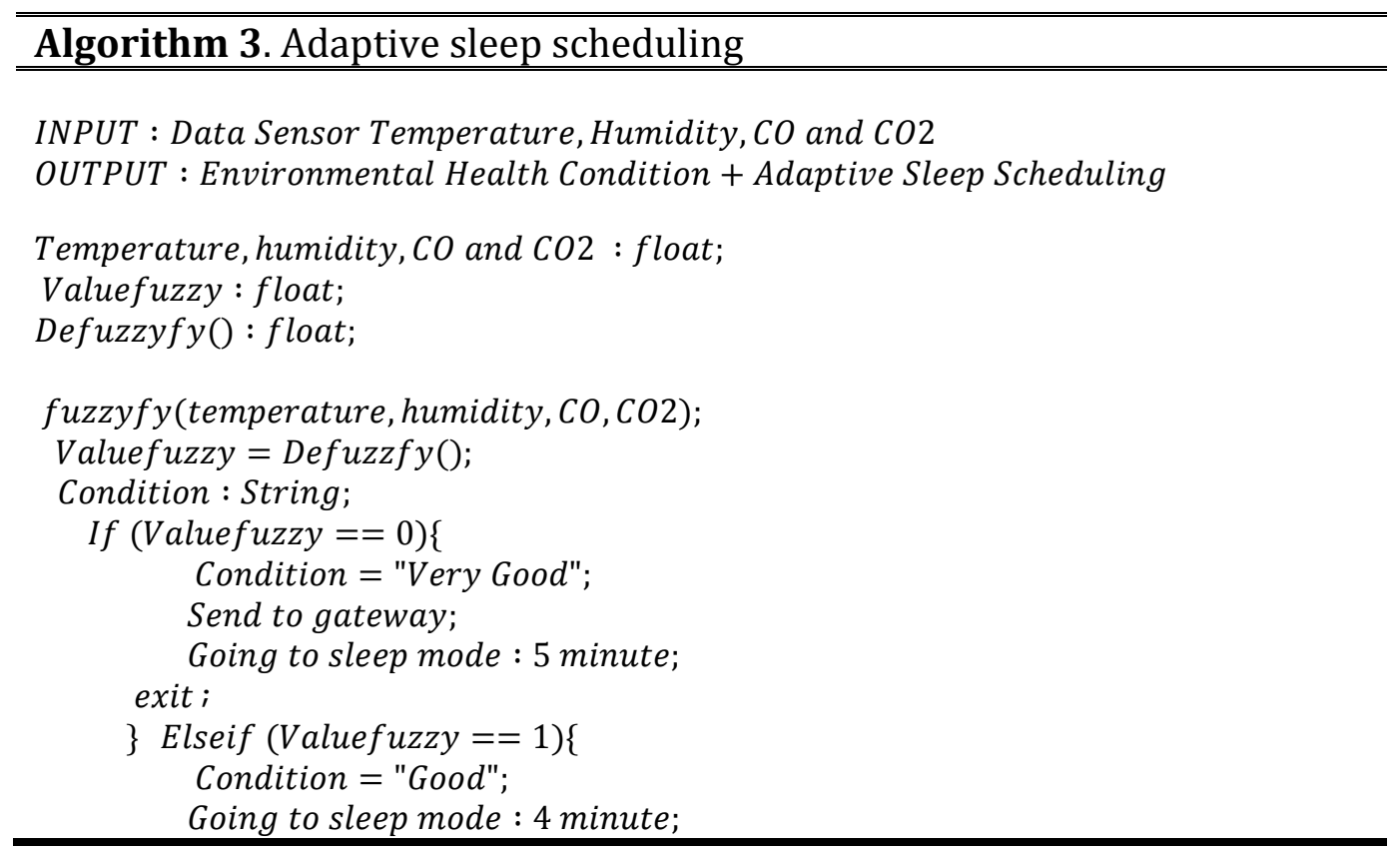




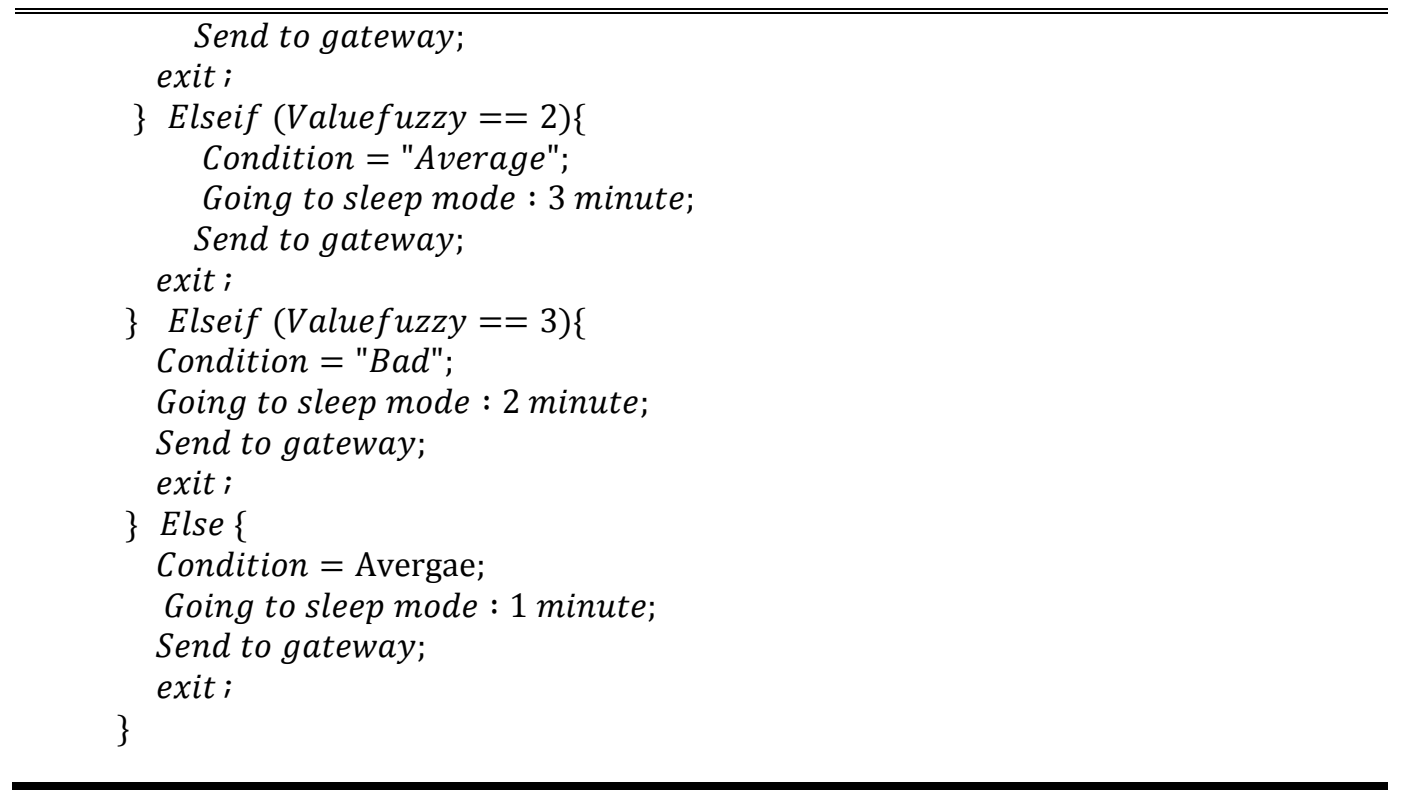

\subsection{Data Sensor Synchronization}

For the gateway, we use Meshlium plug \& sense which has a role as the internet gateway for waspmote. It is provided by libelium [17]. The operating system used by waspmote is Linux based mesh router. It is consist of 5 radios such as WiFi 2,4 GHz, WiFi $5 \mathrm{GHz}$, ZigBee, Bluetooth and 3G/GPRS. This system also provide the Geode Integrated AMD PCS x86 with Processor $500 \mathrm{MHz}$, cache memory 128KB, RAM 256MB, Disk 8GB, Linux Debian kernel2.6.30, WiFi Atheros AR5213A 802.11b/g 100mW -20dBm, XBee Pro 802.15.4 2.4GHz 100mW, and Ethernet Controller VIA VT6105M.

The data from the sensor node is transmitted to the gateway through ZigBee 802.15.4 protocol. All data from sensor node are saved to the database storage temporarily wherein the gateway, MySQL database is provided. Furthermore, the sensor data are collected in the gateway and synchronized to Data Center Server via TCP / IP for storing permanently.

\subsection{Data Sensor Access}

DCS which is used in this experiment is a Control Processor Unit (CPU) as servers on the board with ARM processor $2.60 \mathrm{GHz}$ clock speed. The CPU performance uses core i5 with Read Access Memory (RAM) 4 GB and the storage capacity provided 500GB. In this system, we use Operating System, Mac OS X with 64bit and we install XAMPP 5.0 as database center. Internet Browser used in this experiment are Mozilla Firefox and Google Chrome. These Internet browsers are used for monitoring via a web-based application. Sensor node will send the data to the gateway and the gateway will store the data that have been provided and synchronized by that gateway to DCS through Ethernet LAN/IP. This system must match the IP and database name and default port from XAMPP and all sensor data will be stored permanently. All users can access data sensor via web-based 
application or mobile phone anywhere and anytime as long as connected to the Internet.

\section{EXPERIMENT AND ANALYSIS}

In this section, we explain the experiment and analysis of our WSN energy efficient based on IEEE 802.15.4 standard for environmental health monitoring with FLC approach. Table 2 shows software specifications and hardware which is used in the experiment.

Table 2. Hardware and software specifications used in the experimental.

\begin{tabular}{|c|c|c|}
\hline $\begin{array}{c}\text { Sensor Nodes } \\
(\text { Node1 Node } 3)\end{array}$ & Gateway & Data Center Server \\
\hline $\begin{array}{l}\text { Microcontroller } \\
\text { ATmega1281 } \\
\text { 14MHz, SRAM 8KB, } \\
\text { EEPROM 4KB, } \\
\text { FLASH 128KB, } \\
\text { Clock RTC } 32 \mathrm{KHz}, \\
\text { 802.15.4/ZigBee } \\
2.4 \mathrm{GHz}\end{array}$ & 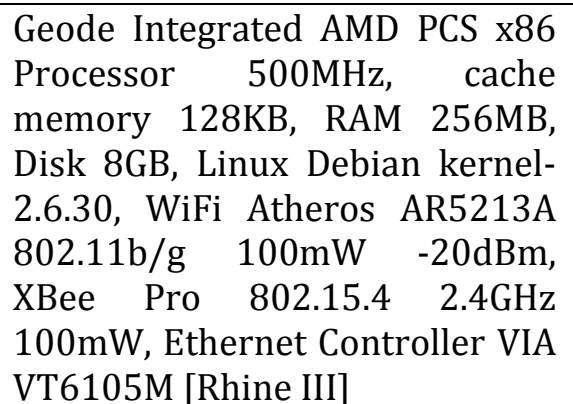 & $\begin{array}{l}\text { Mac OS X } 10.11 .3 \text {, } \\
\text { Processor 2.5 GHz } \text { Intel } \\
\text { Core i5, RAM } 4 \text { GB } 1600 \\
\text { MHz DDR3, HDD 500GB, } \\
\text { XAMPP 5.0, Chrome, PHP, } \\
\text { database MySQL. }\end{array}$ \\
\hline
\end{tabular}

In this paper, we implement the environmental health monitoring on the highway road in Surabaya city. We use three sensor nodes, gateways, and Data Center Server (DCS). Each sensor node consists of four sensors such as temperature, humidity, $\mathrm{CO}$ and $\mathrm{CO} 2$ sensor, as well as a 3.7-volt battery with a capacity of $1150 \mathrm{mAh}$ that serves to turn on the sensor nodes. Gateway used for temporary storage of the sensor node. The gateway has limited data storage capacity [17]. And the last is the DCS which serves as a permanent storage, which the result from synchronizing from the gateway. Users can access the sensor data in the DCS via web-based and / or smartphones such as figure 8-9. 


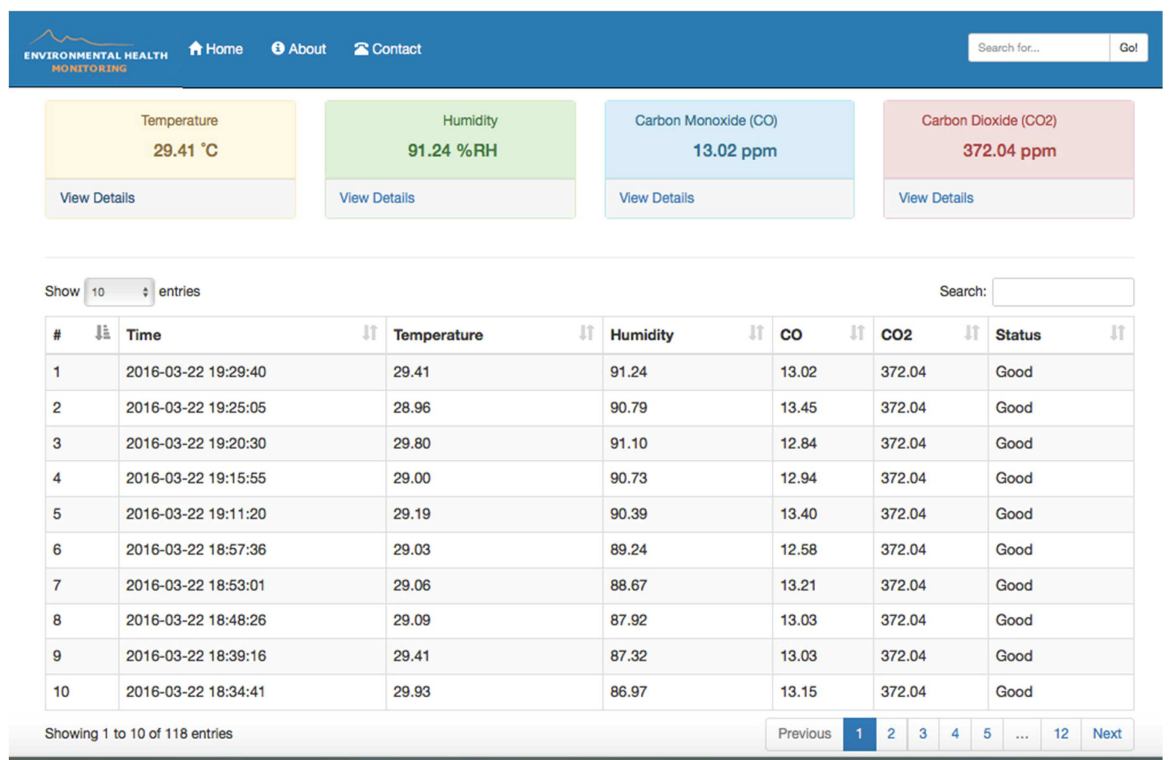

Figure 8. Data sensors access sensor via web-based application

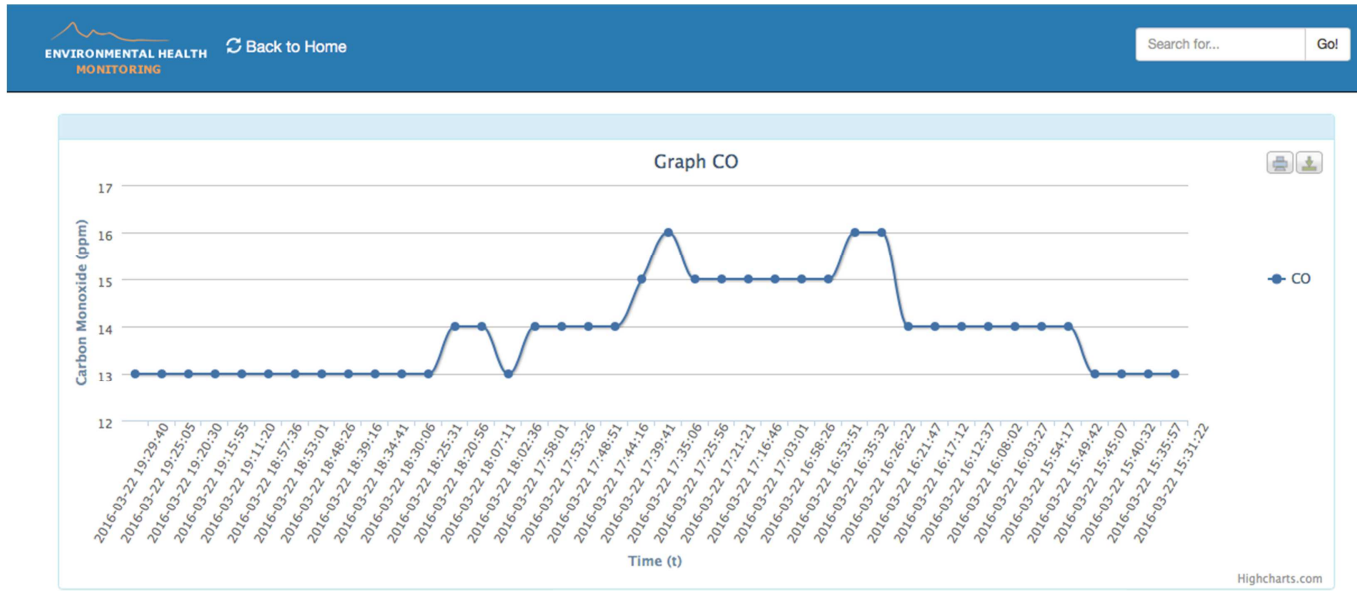

Figure 9. Visualization data sensor from web-based application

Table 3. Data sample of environmental health condition using fuzzy logic

\begin{tabular}{c|ccccc}
\hline Time & $\begin{array}{c}\text { Temperature } \\
\left({ }^{\circ} \mathrm{C}\right)\end{array}$ & $\begin{array}{c}\text { Humidity } \\
(\% \mathrm{RH})\end{array}$ & $\begin{array}{c}\mathrm{CO} \\
(\mathrm{ppm})\end{array}$ & $\begin{array}{c}\text { CO2 } \\
(\mathrm{ppm})\end{array}$ & $\begin{array}{c}\text { Environmental } \\
\text { Health } \\
\text { Condition }\end{array}$ \\
\hline 09:00 & 32.35 & 80.48 & 18.48 & 372.94 & Good \\
$09: 30$ & 33.41 & 73.56 & 19.38 & 372.15 & Good \\
$10: 00$ & 33.16 & 72.73 & 18.73 & 390.04 & Good \\
$10: 30$ & 33.19 & 72.73 & 17.62 & 389.36 & Good \\
$11: 00$ & 35.54 & 66.45 & 19.55 & 400.87 & Good \\
$11: 30$ & 35.09 & 60.32 & 20.02 & 402.17 & Good \\
$12: 00$ & 34.87 & 62.64 & 17.33 & 396.93 & Good \\
$12: 30$ & 33.93 & 75.87 & 16.75 & 390.13 & Good \\
$13: 00$ & 34.03 & 73.77 & 17.48 & 389.67 & Good \\
$13: 30$ & 33.37 & 70.03 & 17.67 & 392.56 & Good \\
\hline
\end{tabular}




\begin{tabular}{|l|lllll|}
\hline 14:00 & 32.61 & 75.80 & 15.21 & 400.67 & Good \\
\hline $14: 30$ & 30.87 & 80.14 & 14.01 & 394.34 & Good \\
$15: 00$ & 31.22 & 81.11 & 14.62 & 378.23 & Good \\
15:30 & 29.93 & 80.93 & 13.22 & 379.09 & Good \\
$16: 00$ & 30.80 & 81.46 & 14.07 & 379.23 & Good \\
$16: 30$ & 31.22 & 80.40 & 16.29 & 383.42 & Good \\
$17: 00$ & 31.25 & 81.23 & 14.91 & 392.12 & Good \\
$17: 30$ & 30.19 & 81.96 & 16.13 & 367.04 & Good \\
$18: 00$ & 30.09 & 83.12 & 13.34 & 368.37 & Good \\
$18: 30$ & 29.41 & 86.99 & 13.11 & 372.04 & Good \\
$19: 00$ & 29.03 & 89.24 & 12.58 & 334.57 & Good \\
$19: 30$ & 29.41 & 91.24 & 13.02 & 334.57 & Good \\
\hline
\end{tabular}

In the experiment, sensor nodes will measure the environmental health condition monitoring system based on WSN with fuzzy logic as decision making about environmental conditions based on the measurement of sensor data. In this system, we collect sensor data such as temperature, humidity, CO and $\mathrm{CO} 2$ from the sensor nodes. The system automatically assigns the decision of the measurement results from data sensor in the form the warnings, those are Very Good (VG), Good (G), Average (A), Bad (B) and Dangerous (D).

Table 3 shows the results of measurements for environmental health condition by using fuzzy logic approach. We take measurements from 09:00 am until 19:30 pm. For the example at 09:00 am, the sensor data produced the temperature $=32.35^{\circ} \mathrm{C}$, humidity $=80.48 \% \mathrm{RH}, \mathrm{CO}=18.48$, and $\mathrm{CO} 2=$ 372.94 then the decision of fuzzy for the current health condition is Good (G). In this experiment of the measurement in all conditions we get is Good (G). But, there are differences in the conditions of temperature, humidity, $\mathrm{CO}$ and CO2 measurements every time. For example, at 11:00 (temperature $=35.54$ ${ }^{\circ} \mathrm{C}$, humidity $=66.45 \% \mathrm{RH}, \mathrm{CO}=19.55 \mathrm{ppm}$ and $\mathrm{CO} 2=400.87 \mathrm{ppm}$ ) there are differences in the measurement results from the previous hour at 10:30 (temperature $=33.19^{\circ} \mathrm{C}$, humidity $=72.73 \% \mathrm{RH}, \mathrm{CO}=17.62 \mathrm{ppm}$ and $\mathrm{CO} 2=$ $389.36 \mathrm{ppm}$ ). This is due at 11:00 until 14:00 pm many human activities such as motorcycles, cars and so forth so as to increase the environmental health condition on the highway, such as temperature, humidity, $\mathrm{CO}$ and $\mathrm{CO} 2$, but this condition is still not dangerously or bad for humans because of the conditions at the time this is still categorized Good (G). Figure 10 shown the graph sensor temperature, humidity, $\mathrm{CO}$ and $\mathrm{CO} 2$ from the measurement of environmental health conditions. 


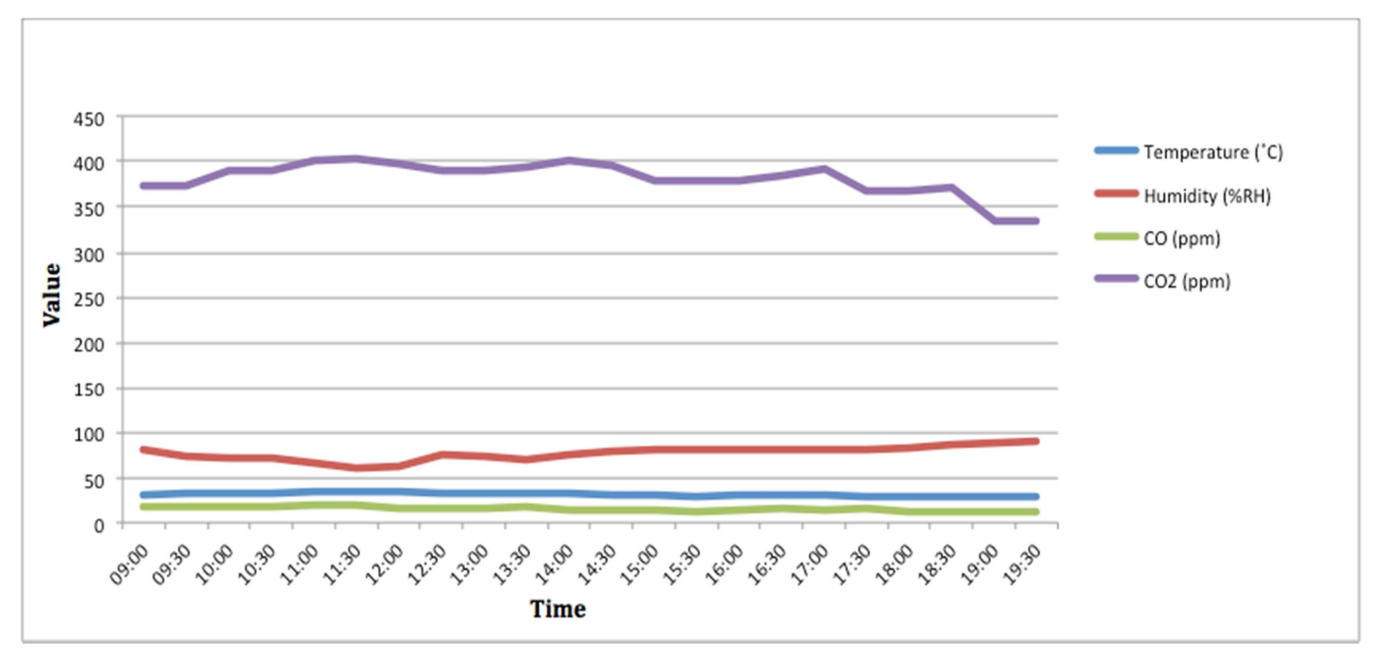

Figure 10. Data sensor temperature, humidity, $\mathrm{CO}$ and $\mathrm{CO} 2$

From the measurement results of the environmental health conditions by using the fuzzy logic approach as shown in Table 3, we propose a process for transmitting data to the gateway based on the output fuzzy logic based on environmental health conditions which are aimed to save energy battery. Here, we compare three processes to save energy battery, namely without sleep scheduling, with sleep scheduling and adaptive sleep scheduling.

Without sleep scheduling: In this system, sensor node will capture data and sending data to the gateway continuously. So the power consumption of battery will be quickly exhausted like table 4 shows a power consumption battery without sleep scheduling.

Table 4. Power consumption battery without sleep scheduling

\begin{tabular}{|c|ccc|}
\hline No & Time & $\begin{array}{c}\text { Battery } \\
\text { Level (\%) }\end{array}$ & $\begin{array}{c}\text { Battery } \\
\text { Voltage (volt) }\end{array}$ \\
\hline 1 & $09: 00$ & 87 & 4.141027 \\
& $10: 00$ & 79 & 4.055611 \\
2 & $10: 02$ & 79 & 4.055611 \\
& $11: 00$ & 69 & 3.948841 \\
3 & $11: 01$ & 69 & 3.948841 \\
& $12: 00$ & 63 & 3.884779 \\
4 & $12: 01$ & 63 & 3.884779 \\
\hline 5 & $13: 00$ & 58 & 3.831394 \\
& $13: 01$ & 58 & 3.831394 \\
6 & $14: 00$ & 50 & 3.745978 \\
\hline 7 & $14: 01$ & 50 & 3.745978 \\
& $15: 00$ & 44 & 3.681916 \\
\hline 9 & $15: 01$ & 44 & 3.681916 \\
\hline & $16: 00$ & 41 & 3.649885 \\
& $16: 01$ & 41 & 3.649885 \\
\hline
\end{tabular}




\begin{tabular}{|l|lll|}
\hline \multirow{3}{*}{10} & $18: 00$ & 38 & 3.627464 \\
\cline { 2 - 4 } & $18: 01$ & 38 & 3.627464 \\
\cline { 2 - 4 } & $19: 00$ & 35 & 3.595433 \\
\hline
\end{tabular}

From the literature [17], it explained that the maximum power when the battery is fully charged with a level of the 4.2 -volt battery is $100 \%$. In this experiment, the initial battery condition in the process of data sensing is $87 \%$ and the final condition is $35 \%$. So that there is a decrease in battery level around $52 \%$. Figure 11 shows the battery power consumption without sleep scheduling.

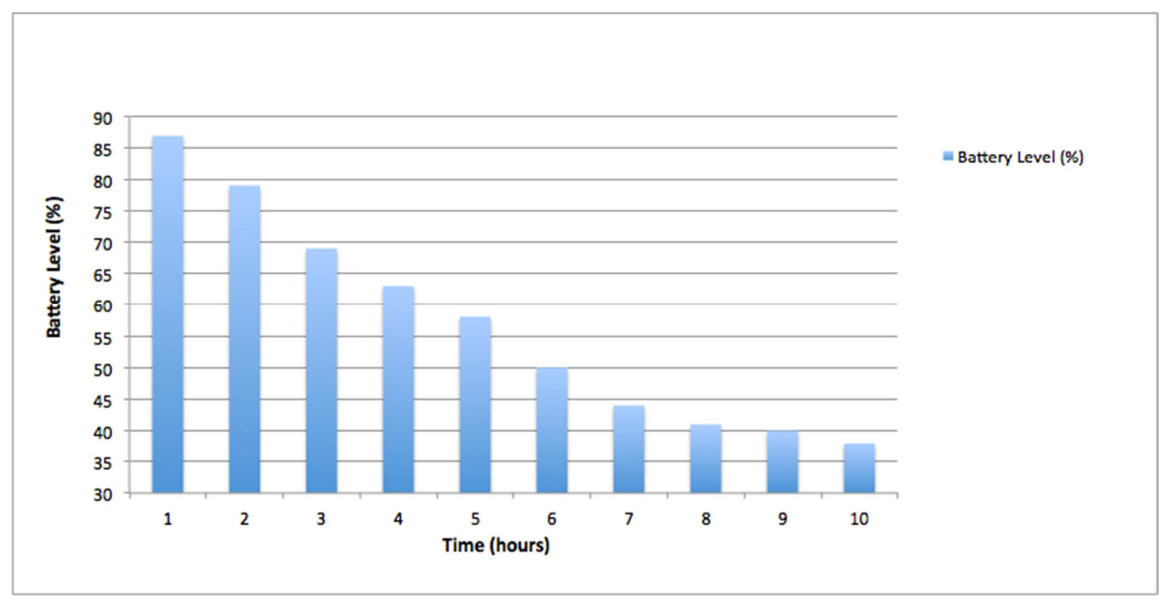

Figure 11. Power consumption of battery without sleep scheduling

The initial of the battery voltage conditions at the beginning of data sending is 4.141027 volts and the final condition is 3.595433 volts. So that there is a decrease on battery voltage consumption around 0.545594 volts. Figure 12 shows the graph of battery voltage without sleep scheduling.

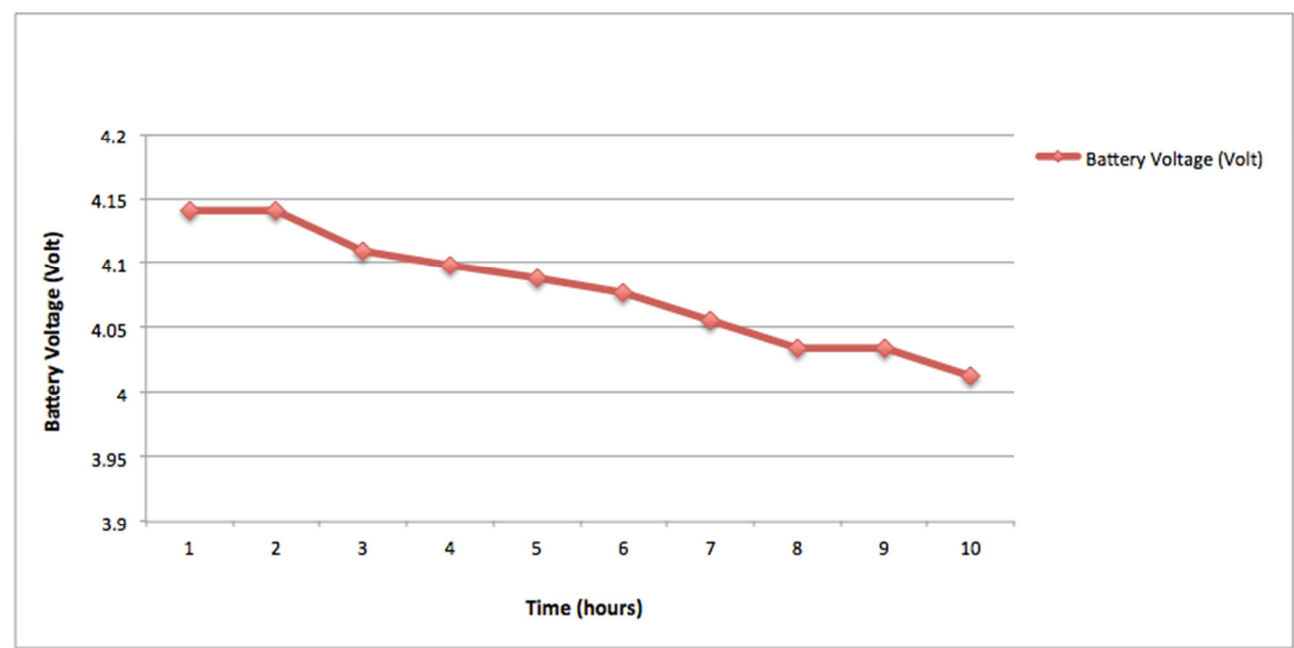

Figure 12. Consumption of battery voltage without sleep scheduling 
With sleep scheduling: In this system, sensor node will send data to the gateway by periodically, we set time to transmit the data every 2 minutes and after that Microcontroller Control Unit (MCU) and Gases Board will soon go to sleep. In this case, we use a hibernating process, because in this system [17], usage of power consumption when board condition is $\mathrm{ON}$ is around $9 \mathrm{~mA}$, Sleep $62 \mu \mathrm{A}$, Deep Sleep $62 \mu \mathrm{A}$, and the last is Hibernate, the consumption is only $0.6 \mu \mathrm{A}$. In the hibernate process, this mode decreases lower consumption without disconnection to the module. Waspmote board, gasses board and ZigBee will be off except the Read Time Clock (RTC). RTC only be used to set time wake up from the hibernating mode. Table 5 shows a battery power consumption with sleep scheduling.

Table 5. Power consumption battery with sleep scheduling

\begin{tabular}{|c|c|c|c|}
\hline No & Time & $\begin{array}{c}\text { Battery } \\
\text { Level (\%) }\end{array}$ & $\begin{array}{c}\text { Battery } \\
\text { Voltage (volt) }\end{array}$ \\
\hline \multirow[t]{2}{*}{1} & 09:00 & 87 & 4.141027 \\
\hline & $10: 00$ & 86 & 4.130350 \\
\hline \multirow[t]{2}{*}{2} & $10: 02$ & 86 & 4.130350 \\
\hline & $11: 00$ & 84 & 4.108996 \\
\hline \multirow[t]{2}{*}{3} & 11:01 & 84 & 4.108996 \\
\hline & $12: 00$ & 83 & 4.098319 \\
\hline \multirow[t]{2}{*}{4} & $12: 01$ & 83 & 4.098319 \\
\hline & $13: 00$ & 82 & 4.087642 \\
\hline \multirow[t]{2}{*}{5} & 13:01 & 82 & 4.087642 \\
\hline & $14: 00$ & 81 & 4.076965 \\
\hline \multirow[t]{2}{*}{6} & $14: 01$ & 81 & 4.076965 \\
\hline & $15: 00$ & 79 & 4.055611 \\
\hline \multirow[t]{2}{*}{7} & $15: 01$ & 79 & 4.055611 \\
\hline & $16: 00$ & 78 & 4.034257 \\
\hline \multirow[t]{2}{*}{8} & $16: 01$ & 78 & 4.034257 \\
\hline & $17: 00$ & 78 & 4.034257 \\
\hline \multirow[t]{2}{*}{9} & 1701 & 78 & 4.034257 \\
\hline & $18: 00$ & 76 & 4.012903 \\
\hline \multirow[t]{2}{*}{10} & 18:01 & 76 & 4.012903 \\
\hline & $19: 00$ & 74 & 3.991549 \\
\hline
\end{tabular}

With sleep scheduling, the initial battery condition when the process of data sensing is $87 \%$ and the final condition is $74 \%$. So there is a decrease in battery level around 13\%. Figure 13 shows the battery power consumption with sleep scheduling. 


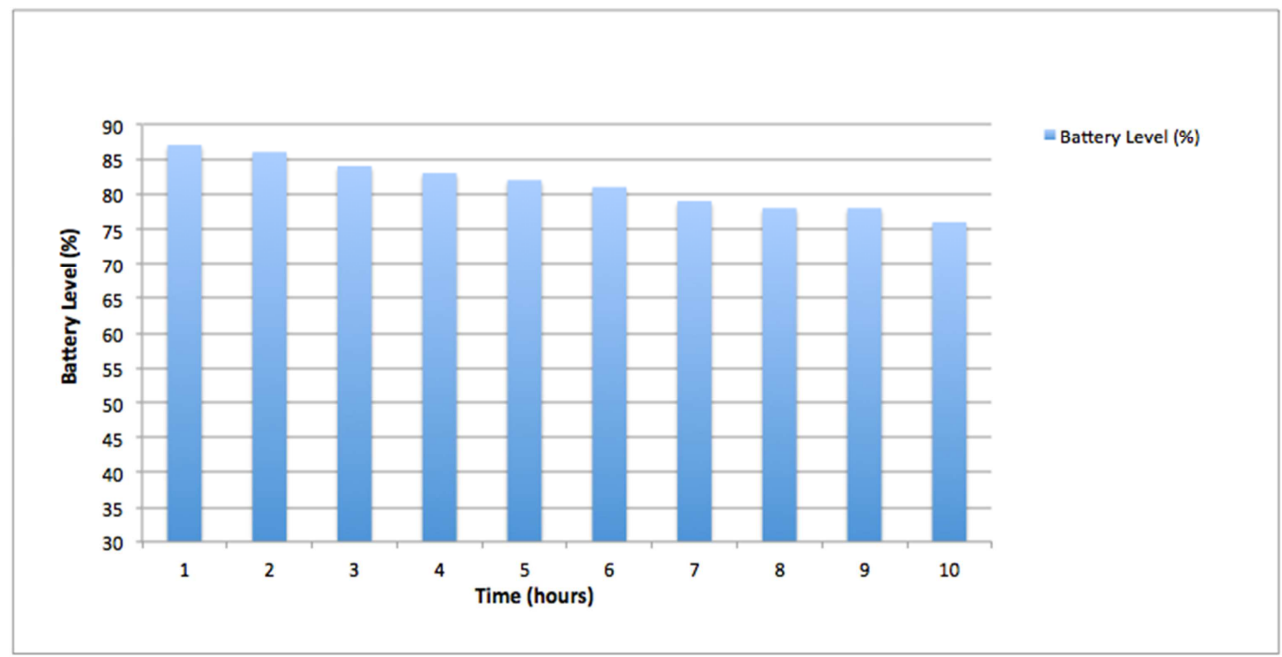

Figure 13. The power consumption of battery with sleep scheduling

The initial of the battery voltage conditions when the process of sending data begins is 4.141027 volts and the final condition is 3.991549 volts. So that there is a decrease of battery voltage around 0.149478 volts. Figure 14 shows the graph battery voltage with sleep scheduling.

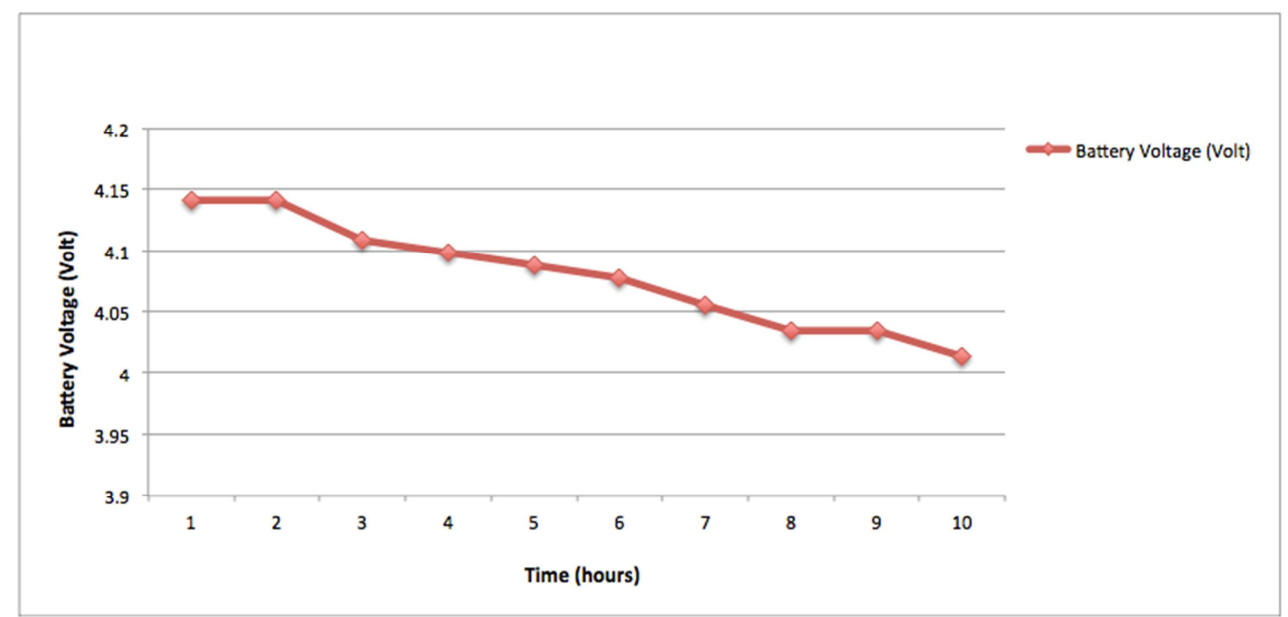

Figure 14. Consumption of battery voltage with sleep scheduling

Adaptive sleep scheduling: In this process, adaptive transmission control is based on the fuzzy logic approach which the result is a combination of each sensor, consists of temperature, humidity, carbon monoxide (CO) and carbon dioxide (CO2). The output of the resulting fuzzy logic is classified as follows: Very Good (VG), Good (G), Average (A), Bad, and Dangerous (D). We used the output that is resulted from fuzzy logic as transmission of adaptive process, in which this system will set up the time of data transmission for the environmental health condition base on fuzzy value. If the condition is Very Good (VG), then the sensor node will send data to the gateway for every 5 minutes periodically and all the components will soon sleep except RTC. Meanwhile, if the condition is Good (G), node sensor will send data to the 
gateway for every 4 minutes and until the conditions are considered as Dangerous (D), where the sensor node will transmit data for every 1 minute. Table 6 shows the battery power consumption with adaptive sleep scheduling.

Table 6. Power consumption battery with adaptive sleep scheduling

\begin{tabular}{|c|c|c|c|}
\hline No & Time & $\begin{array}{c}\text { Battery } \\
\text { Level (\%) }\end{array}$ & $\begin{array}{c}\text { Battery } \\
\text { Voltage (volt) }\end{array}$ \\
\hline \multirow[t]{2}{*}{1} & 09:00 & 87 & 4.141027 \\
\hline & $10: 00$ & 86 & 4.130350 \\
\hline \multirow[t]{2}{*}{2} & $10: 02$ & 86 & 4.130350 \\
\hline & $11: 00$ & 86 & 4.130350 \\
\hline \multirow[t]{2}{*}{3} & $11: 01$ & 86 & 4.130350 \\
\hline & $12: 00$ & 85 & 4.119673 \\
\hline \multirow[t]{2}{*}{4} & $12: 01$ & 85 & 4.119673 \\
\hline & $13: 00$ & 84 & 4.108996 \\
\hline \multirow[t]{2}{*}{5} & $13: 01$ & 84 & 4.108996 \\
\hline & $14: 00$ & 83 & 4.098319 \\
\hline \multirow[t]{2}{*}{6} & $14: 01$ & 83 & 4.098319 \\
\hline & $15: 00$ & 83 & 4.098319 \\
\hline \multirow[t]{2}{*}{7} & $15: 01$ & 83 & 4.098319 \\
\hline & $16: 00$ & 82 & 4.087642 \\
\hline \multirow[t]{2}{*}{8} & $16: 01$ & 82 & 4.087642 \\
\hline & $17: 00$ & 82 & 4.087642 \\
\hline \multirow[t]{2}{*}{9} & 1701 & 82 & 4.087642 \\
\hline & $18: 00$ & 81 & 4.076965 \\
\hline \multirow[t]{2}{*}{10} & $18: 01$ & 81 & 4.076965 \\
\hline & 19:00 & 80 & 4.066288 \\
\hline
\end{tabular}

With adaptive sleep scheduling, the initial battery condition when the process of sending data is $87 \%$ and the final condition is $80 \%$. So that there is a decrease in battery level around 7\%. Figure 15 shows the power consumption battery with adaptive sleep scheduling.

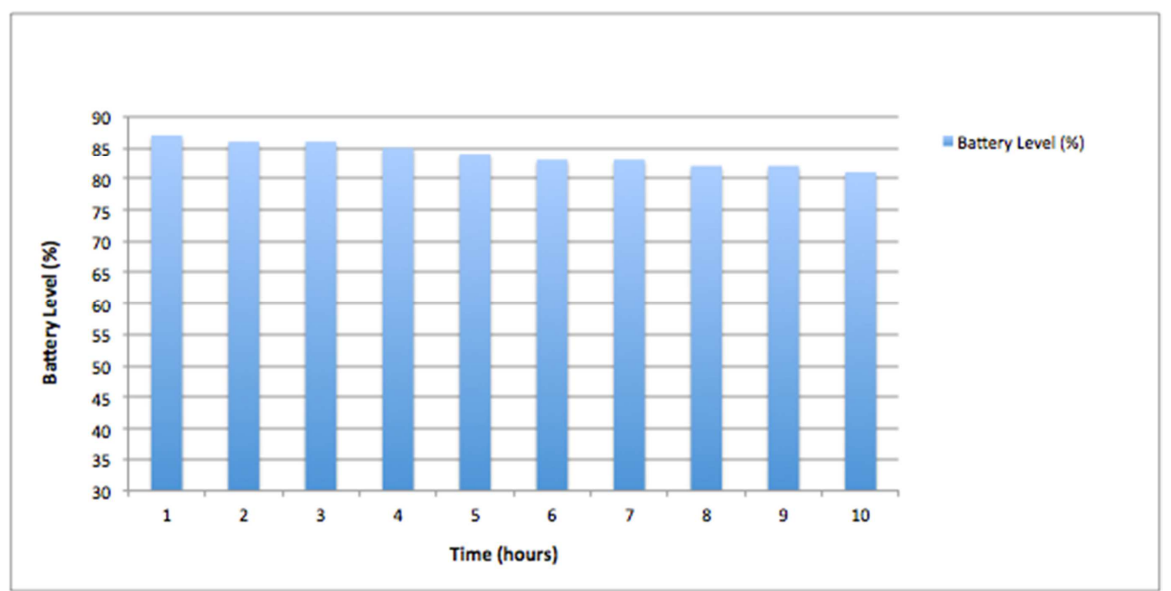

Figure 15. Power consumption battery with adaptive sleep scheduling 
The initial conditions of the battery voltage when the process of sending data begins is 4.141027 volts and the final condition is 4.066288 volts. So there is a decrease of battery voltage around 0.074739 volts. Figure 16 shows the graph battery voltage with sleep scheduling.

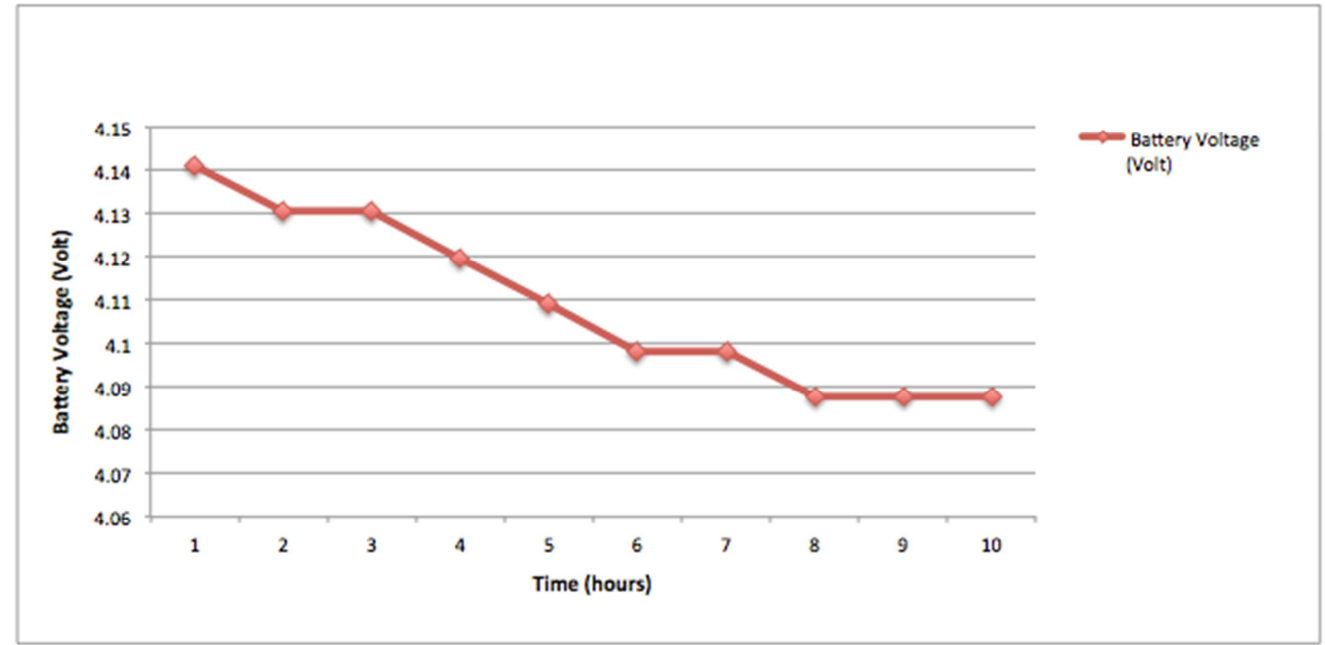

Figure 16. Consumption of battery voltage with adaptive sleep scheduling

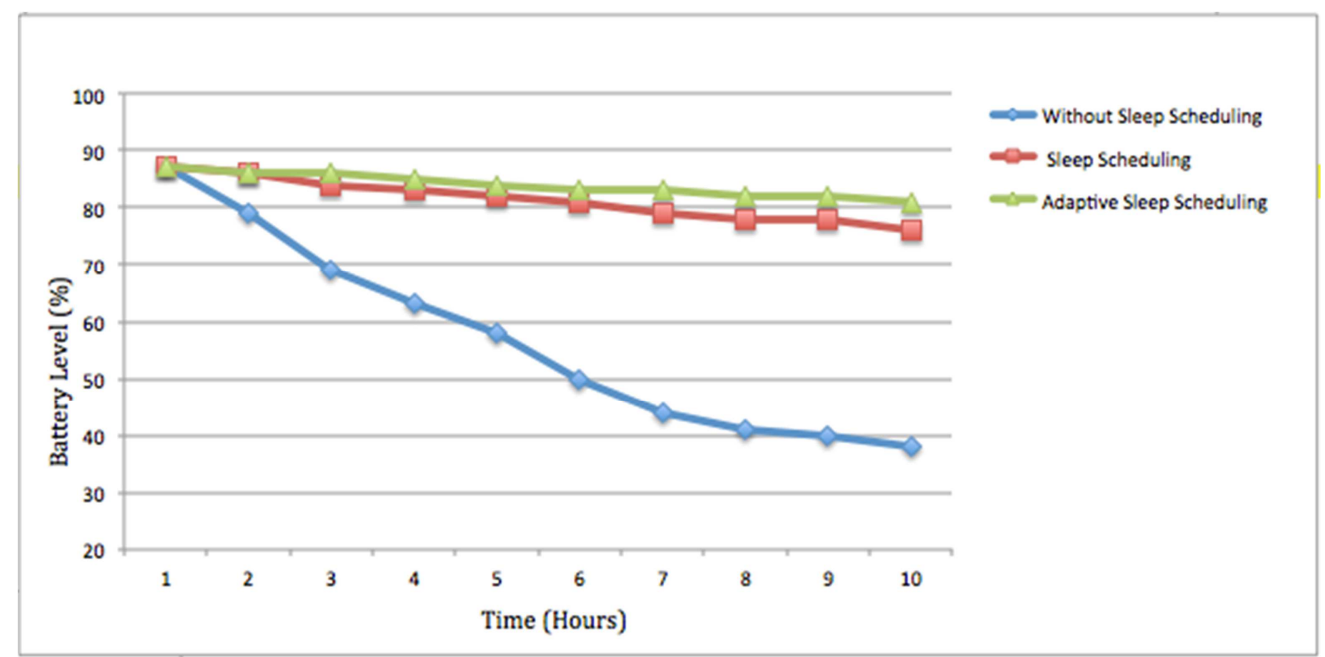

Figure 17. Energy consumption battery level without sleep scheduling, sleep scheduling, and adaptive sleep scheduling

From the described graph above, it is shown that the power consumption of battery dropped dramatically when the process is transmitting data without sleep scheduling if compared to using a sleep scheduling and adaptive sleep scheduling such as shown figure 19. From obtained data in the above, the power consumption spent during the process of testing without sleep scheduling is $52 \%$, for the sleep scheduling is $13 \%$ while using the adaptive sleep scheduling around $7 \%$. 


\section{CONCLUSION}

In this paper, we propose an environmental health monitoring system based on IEEE 802.15.4 standard for giving information to humans about the environmental health condition. This system uses various sensors such as temperature, humidity, carbon monoxide ( $\mathrm{CO}$ ) and carbon dioxide (CO2). Furthermore, our paper uses the fuzzy logic approach as making a decision based on the combination of multiple sensors. To improve the energy efficiency, we use an adaptive sleep scheduling based on the fuzzy logic output. This sleep scheduling is aimed to extend the sensor node lifetime. We also implemented this proposed system in real hardware and analyze the used power consumption by each node. In this experiment, the results are obtained by comparing three transmission processes. The proposed adaptive sleep scheduling significantly reduces the power consumption of WSN. We also provide the system to monitor the condition of environmental health in real-time condition, where all users can access using their smartphone and/or web-based application anywhere and anytime.

\section{REFERENCES}

[1] Sheikh Ferdoush, Xinrong Li, Wireless Sensor Network System Design using Raspberry $\mathrm{Pi}$ and Arduino for Environmental Monitoring Applications, The 9th International Conference on Future Networks and Communications (FNC-2014), Niagara Falls, Canada, pp. 103-110, 2014.

[2] M.F Othmana, K. Shazalib, Wireless Sensor Network Applications: A Study in Environment Monitoring System, International Symposium on Robotics and Intelligent Sensors 2012 (IRIS 2012), ScienceDirect, pp. 1204-1210, 2012.

[3] Dunfan Ye, Daoli Gong, Wei Wang, Application of Wireless Sensor Networks in Environmental Monitoring, Power Electronics and Intelligent Transportation System (PEITS), Shenzhen, pp. 205 - 208, 2009.

[4] S. Abraham, Xinrong Li, A Cost-Effective Wireless Sensor Network System for Indoor Air Quality Monitoring Applications, Future Networks and Communications (FNC-2014), ScienceDirect, pp. 165-171, 2014.

[5] Octavian A. Postolache, J. M. Dias Pereira, and P. M. B. Silva Girao, Smart Sensors Network for Air Quality Monitoring Applications, IEEE Transactions on Instrumentation and Measurement, Vol. 58, pp. 3253 3262, 2009.

[6] M. Dutta, S. Bhowmik, and C. Giri, Fuzzy Logic Based Implementation for Forest Fire Detection Using Wireless Sensor Network, Smart Innovation, Systems and Technologies, Switzerland, pp. 319-327, 2014

[7] N. Fahmi, S. Huda, M.U.H Al Rasyid, A. Sudarsono, Fuzzy Logic for an Implementation Environment Health Monitoring Based on Wireless Sensor Network, Recent Advancement in Informatics, Electrical and 
Electronics Engineering International Conference (RAIEIC 2015), Medan, 2015.

[8] M.R Tripathy, K. Gaur, S Sharma, and G.S. Virdi, Energy Efficient Fuzzy Logic Based Intelligent Wireless Sensor Network, Progress In Electromagnetics Research Symposium Proceedings, Cambridge, USA, pp. 91-95, 2010.

[9] Giovanni Pau, Power Consumption Reduction for Wireless Sensor Networks Using A Fuzzy Approach, International Journal of Engineering and Technology Innovation (IJETI), Taiwan, 2015.

[10] R. Sabitha, K.T. Bhuma, and T. Thyagarajan, Design and Analysis of Fuzzy Logic and Neural Network Based Transmission Power Control Techniques for Energy Efficient Wireless Sensor Networks, Advances in Intelligent Systems and Computing, Springer International Publishing, V0l.1, pp. 295-303, 2015.

[11] A. Sudarsono, P. Kristalina, M.U.H Al Rasyid, R. Hermawan, An implementation of secure data sensor transmission in Wireless Sensor Network for monitoring environmental health, Computer, Control, Informatics and its Applications (IC3INA), Bandung, pp. 93 - 98, 2014.

[12] Jang, J.S.R C.T. Sun dan E. Mizutani. 1997. Neuro-Fuzzy and Soft Computing. London : Prentice-Hall.

[13] P. Patil, U. Kulkarni, B.L. Desai,V.I. Benagi and V.B. Naragund, Fuzzy Logic Based Irrigation Control Using Wireless Sensor Network for Precision Agriculture, AIPA, India, pp. 262-269, 2012

[14] Waspmote Specification - Spesifikasi hardware waspmote. https://www.libelium.com/products/waspmote/hardware/ [accessed on April 2016].

[15] Libelium - Gases 2.0 Technical Guide. https://www.libelium.com/ [accesed on April 2016].

[16] Meshlulim - Meshlium Datasheet and documentation, http://www.libelium.com/development/meshlium/documentation/ [accesed on April 2016].

[17] Libelium - Waspmote Power Programming Guide, http://www.libelium.com/development/waspmote/documentation/w aspmote_power_programming_guide/[accesed on April 2016]. 